\title{
openheart DuraGraft vascular conduit preservation solution in patients undergoing coronary artery bypass grafting: rationale and design of a within-patient randomised multicentre trial
}

\author{
Walid Ben Ali, ${ }^{1}$ Pierre Voisine, ${ }^{2}$ Peter Skov Olsen, ${ }^{3}$ Hugues Jeanmart, ${ }^{4}$ \\ Nicolas Noiseux, ${ }^{1}$ Tracy Goeken, ${ }^{5}$ Vilas Satishchandran, ${ }^{5}$ Filippo Cademartiri, ${ }^{6}$ \\ Garry Cutter, ${ }^{7}$ Dave Veerasingam, ${ }^{8}$ Craig Brown, ${ }^{9}$ Maximilian Y Emmert, ${ }^{10}$ \\ Louis P Perrault ${ }^{1}$
}

\begin{abstract}
- Additional material is published online only. To view please visit the journal online (http://dx.doi.org/10.1136/ openhrt-2018-000780).
\end{abstract}

To cite: Ben Ali W, Voisine $P$ Olsen PS, et al. DuraGraft vascular conduit preservation solution in patients undergoing coronary artery bypass grafting: rationale and design of a withinpatient randomised multicentre trial. Open Heart

2018;5:e000780. doi:10.1136/ openhrt-2018-000780

Received 13 January 2018 Revised 21 February 2018 Accepted 6 March 2018

\section{Check for updates}

For numbered affiliations see end of article.

Correspondence to

Professor Louis P Perrault; Iouis. perrault@icm-mhi.org

\section{ABSTRACT}

Introduction Saphenous vein grafts (SVGs) remain the most often used conduits in coronary artery bypass grafting (CABG). However, they are prone to vein graft disease (VGD) during follow-up, which may compromise clinical outcomes. Injury to the SVG endothelium during harvesting and storage promotes neointimal hyperplasia that can advance to atherosclerosis characterised by SVG failure. This trial investigates the potential benefit of DuraGraft, a novel, one-time intraoperative graft treatment developed to efficiently protect the structural and functional integrity of the vascular endothelium, on the development and progression of VGD in CABG patients.

Methods and analysis This ongoing prospective randomised, double-blinded multicentre trial (NCT02272582/NCT02774824) includes patients undergoing isolated $\mathrm{CABG}$ requiring at least two SVGs. It compares the impact of DuraGraft, a novel treatment against VGD versus the standard-of-care (SOC; heparinised saline) using a within-patient randomisation (with one SVG treated with DuraGraft and the other treated with SOC). Besides clinical assessments, patients undergo longitudinal 64-slice or better multidetector CT (MDCT) angiography of paired grafts (within each patient) at 4-6 weeks, 3 months and 12 months. Primary endpoints will be the magnitude of change in mean wall thickness and lumen diameter (stenosis) of paired grafts, at 3 and 12 months, respectively. Besides the evaluation of overall safety, longitudinal assessment of each graft (secondary endpoint) is performed in order to obtain insight into graft behaviour after CABG. Enrolment of 119 patients was successfully completed, and analysis of MDCT angiography follow-up is ongoing with the completed analysis becoming available by end of first quarter of 2018.

Ethics and dissemination The regional ethics committees have approved the trial. Results will be submitted for publication.

Clinical trial identifier NCT02272582 and NCT02774824.

\section{Key questions}

What is already known about this subject?

- Saphenous vein grafts (SVGs) are the most often used conduits for coronary artery bypass grafting (CABG). However, their use is hampered by loss of graft patency over time due to vein graft disease (VGD) resulting in graft failure potentially impairing long-term clinical outcomes.

- Damage to the conduit's endothelium during harvest and storage (prior to anastomosis) has been identified as the main trigger for VGD. Importantly, standard intraoperative solutions (ie, saline or ex vivo blood) are not able to sufficiently protect the conduit's endothelium thereby promoting failure and poor long-term patency.

What does this study add?

- This trial will establish whether pretreatment of SVGs with DuraGraft, an endothelial damage inhibitor, has the potential to mitigate negative remodelling by reducing intima hyperplasia (early signs of VGD) and graft lumen loss (late signs) in CABG patients.

- It will further provide important insight into the Iongitudinal remodelling behaviour and (patho-) physiology of SVGs post-CABG.

How might this impact on clinical practice?

- Novel graft treatments specifically designed to protect the structural and functional integrity of the conduit's endothelium may substantially reduce the occurrence of VGD thereby further improving longterm outcomes after CABG

\section{INTRODUCTION}

Coronary artery bypass grafting (CABG) is the standard of care for multivessel coronary disease. ${ }^{2}{ }^{2}$ However, saphenous vein graft (SVG) patency loss due to vein graft 
disease (VGD) and consecutive vein graft failure (VGF) remains a major problem resulting in impaired clinical long-term outcomes. ${ }^{3}$ VGF rates have been reported to range from $15 \%$ to $29 \%,{ }^{4}$ while about $50 \%$ of SVGs fail by 10 years post-CABG. ${ }^{36}{ }^{6}$ Long-term graft patency itself is determined by multiple important factors, such as the progress of the patient's coronary artery disease, quality of target vessel, quality of anastomosis, bypass run-off and, most importantly, the quality of the graft itself. Several aspects play a crucial role in preserving the SVG's quality: (1) harvesting techniques to minimise trauma; (2) the impact of hydrostatic pressure during intraoperative distensions when checking for potential leakages and (3) sufficient intraoperative graft storage between harvesting and reperfusion after completion of anastomosis in order to protect the SVG endothelium in its structure and function. ${ }^{3-9}$ In fact, endothelial injury of the endothelial layer promotes leucocyte and platelet adhesion leading to chronic inflammation and an obliterative lesion, which may result in SVG stenosis and finally occlusion. ${ }^{10}$ A number of ex vivo investigations have highlighted the importance of intraoperative preservation of the SVG's endothelium to reduce the problem of VGF. ${ }^{11-15}$ More recently, a substudy of the PREVENT-IV trial further demonstrated that neither saline nor autologous blood (although being the most commonly used solutions) are capable to sufficiently preserve the SVG endothelium in regard to its structure and function during ischaemic storage. ${ }^{6}$ Hence, there is a clinical need for improved intraoperative SVG preservation strategies.

In this context, DuraGraft is one of the most widely recognised solutions for the intraoperative treatment and preservation of the SVG's endothelium during ischaemic storage. DuraGraft is based on a physiological salt solution and further contains glutathione and L-ascorbic acid, antioxidants and arginine, a substrate for nitric oxide synthase in endothelial cells to systematically protect the endothelium against ischaemic injury during storage. In several ex vivo studies, Thatte and colleagues evaluated the effect of DuraGraft against other solutions (ie, saline or blood based solutions) used for storage of SVGs. ${ }^{11}{ }^{16}$ When using multiphoton imaging to elucidate the metabolic changes that take place during intraoperative storage of SVGs, the authors demonstrated that DuraGraft outperformed other solutions such as saline or blood, and it was capable of protecting endothelial structure and function for up to 24 hours of storage. ${ }^{11}$ However, to date, despite these encouraging ex vivo data, systematic data validating the clinical efficacy of DuraGraft to prevent SVGs from the development of intimal hyperplasia leading to VGD and subsequent VGF is still pending.

This trial aims to investigate the potential benefit of DuraGraft, a novel, one-time intraoperative graft treatment developed to efficiently protect the structural and functional integrity of the vascular endothelium on the development and progression of VGD and failure in
CABG patients using longitudinal multidetector CT (MDCT) angiography.

\section{METHODS AND ANALYSIS \\ Trial design}

The trial is designed as a prospective, multicentre, randomised, double-blinded, comparative within-patient study to evaluate the use of DuraGraft, a one-time, intraoperative graft treatment, versus heparin dosed saline (standard of care) in patients for up to 12 months after undergoing isolated CABG.

\section{Trial registration}

The trial is registered at Clinicaltrials.gov under the identifiers: NCT02272582 and NCT02774824.

\section{Objectives}

\section{Primary objectives}

The primary short-term objective is to compare the impact of DuraGraft, a novel, one-time intraoperative graft treatment to protect the structural and functional integrity of the vascular endothelium against the standard of care by evaluating the magnitude of change in the mean wall thickness of paired grafts within patients at 4-6 weeks and at 3 months following CABG surgery using 64-slice or better MDCT angiography. The primary long-term objective is to further compare the effect of DuraGraft against the standard of care by evaluating the effect on graft patency, more precisely by assessing the magnitude of change in a weighted average of mean lumen diameter and lumen diameter at maximum stenosis of paired grafts at 12 months following CABG surgery using 64-slice or better MDCT angiography.

\section{Secondary objectives}

The secondary objectives aim:

- to evaluate the safety of DuraGraft over the 12-month postsurgery period

- to assess composite incidences of major adverse cardiac events (MACEs) (death, myocardial infarction (MI) and repeat revascularisation), angina, arrhythmias and shortness of breath (SOB) over the 12-month postsurgery period.

\section{Patient population}

Eligible patients include those aged between 18 and 80 years undergoing primary and isolated, multivessel CABG requiring at least two SVGs. Patients with previous heart surgery, poor left ventricular ejection fraction, other significant valve disease, need for aortic aneurysm repair, with a stroke or transient ischaemic attack within the previous 12 weeks or any contraindication to cardiopulmonary bypass are excluded. Please see box 1 for full list of all inclusion and exclusion criteria.

In addition, numerous preoperative demographics and characteristics as well as intraoperative parameters and SVG characteristics were recorded (please see online supplementary information for further details). 


\section{Box 1 Inclusion and exclusion criteria}

\section{Inclusion criteria}

Patients will be eligible for inclusion in the study if they meet ALL of the following criteria:

- Patient is to undergo primary, multivessel coronary artery bypass grafting (CABG) with at least two saphenous vein graft (SVGs).

- Patient is $>18$ years and $<75$ years of age.

- Patient has no contraindications to cardiopulmonary bypass.

- Patient is willing and able to provide consent and shows commitment to participate in a follow-up evaluation, including a clinical visit between 4-6 weeks and 3 months post-CABG.

- If female, patient is surgically sterile or postmenopausal.

- Patient has not had previous CABG surgery.

- Patient is hemodynamically stable.

Exclusion criteria

Patients will be excluded from the study if ANY of the following conditions are present:

- in situ internal mammary artery (IMA) graft(s) only (no SVG or free arterial grafts)

- prior CABG or planned concomitant valve surgery or aortic aneurysm repair

- pregnant or lactating woman

- left ventricular ejection fraction $<40 \%$

- known to be HIV positive, is receiving antiretroviral drugs or is immunosuppressed

- patient has an acute infection at screening

- active chronic bacterial, parasitic or viral infection within 3 months prior to CABG surgery

- malignancy diagnosed within the previous 5 years (except successfully resected basal cell cancer)

- unable to provide consent or undergoing emergency cardiac surgery for an immediately life-threatening condition

- participating in a device study or received active drug product in an investigational drug study within 3 months prior to screening

- patient has a history of transient ischaemic attack or stroke within the 12 weeks prior to the CABG procedure

- significant renal impairment (glomerular filtration rate (GFR) $<50 \mathrm{~mL} / \mathrm{min}$ )

- patient has liver impairment as demonstrated by hepatic transaminases (AST and/or ALT) $>2.5 \times$ upper limit of normal (ULN) or conjugated bilirubin $>1.5 \times$ ULN

- any condition or disease detected prior to study start that would render the patient unsuitable for the study place the patient at undue risk or interfere with the ability of the patient to complete the study in the opinion of the investigator (eg, drug dependence and mental illness)

- uncontrolled diabetes mellitus ( $\mathrm{HbA} 1 \mathrm{C}>10 \%)$

- confirmed significant allergic reactions against any drug or multiple allergies (non-active hay fever is acceptable)

- uninterrupted use of systemic steroids or immunosuppressive agents

- platelet count $<100000 / \mathrm{mm}^{3}$, haematocrit $>62 \%(\mathrm{Hb}>18 \mathrm{~g} / \mathrm{dL})$ or $<30 \%(\mathrm{Hb}<10 \mathrm{~g} / \mathrm{L})$

- varicose veins or veins $<2 \mathrm{~mm}$ diameter

- target coronary artery $<1.5 \mathrm{~mm}$ in internal diameter

- diffuse coronary disease

- severe uncontrolled systemic hypertension (ie, systolic pressure $>160 \mathrm{~mm} \mathrm{Hg}$ )

prior severe reaction to contrast dye.

\section{Patient screening}

All patients undergo a screening assessment before enrolment including:

1. review of all inclusion and exclusion criteria to determine eligibility

2. documentation of the medical history including prior medications

3. documentation of demographic information

4. conduct of a 12-lead ECG.

A screening log is maintained at each investigational site to record those patients who are screened but not enrolled and to capture the reason why patients were not enrolled.

\section{Randomisation}

A randomisation schedule was developed to ensure appropriate randomisation allocation of the SVG being grafted to the targeted regions. A dedicated block randomisation schedule was also developed to ensure that at least four African-American patients and four Hispanic patients are enrolled. Patients are randomised using a balanced simple random sample allocation scheme. The randomisation assignments are as follows:

- DuraGraft left proximal (A) - saline right distal (B)

- DuraGraft left distal (A) - saline right proximal (B)

- DuraGraft right proximal (B) - saline left distal (A)

- DuraGraft right distal (B) - saline left proximal (A).

Each randomised patient receives two SVGs, alternating target region A (circumflex or diagonal or other) and target region B (right coronary system or diagonal or other) and alternating (proximal vs distal) segments of the harvested SVG.

\section{Blinding}

Only the personnel who manages the randomisation and allocation schedule will remain unblinded. The surgeon, other staff in the operating room and the personnel collecting the intraoperative data are completely blinded to the preservation and storage solutions used for the two SVGs in each enrolled patient. Since DuraGraft is a transparent solution, it is completely indistinguishable from the standard of care (heparinised saline). The cardiologists assessing clinical events and the radiologists reading the MDCT angiographies are also blinded to the solutions that have been used to preserve and store the SVGs intraoperatively.

\section{Follow-up}

All patients will have a follow-up by a clinic visit at $4-6$ weeks, 3 months and at 12 months after CABG (see table 1 and 2). Each visit will include documentation of concomitant medication information, adverse events, glomerular filtration rate (GFR) and the conduction of an ECG and an MDCT angiography. If a patient withdraws prior to completion of the study, the reason for this decision will be recorded in the case report form (CRF). Whenever possible, all end-of-study procedures 


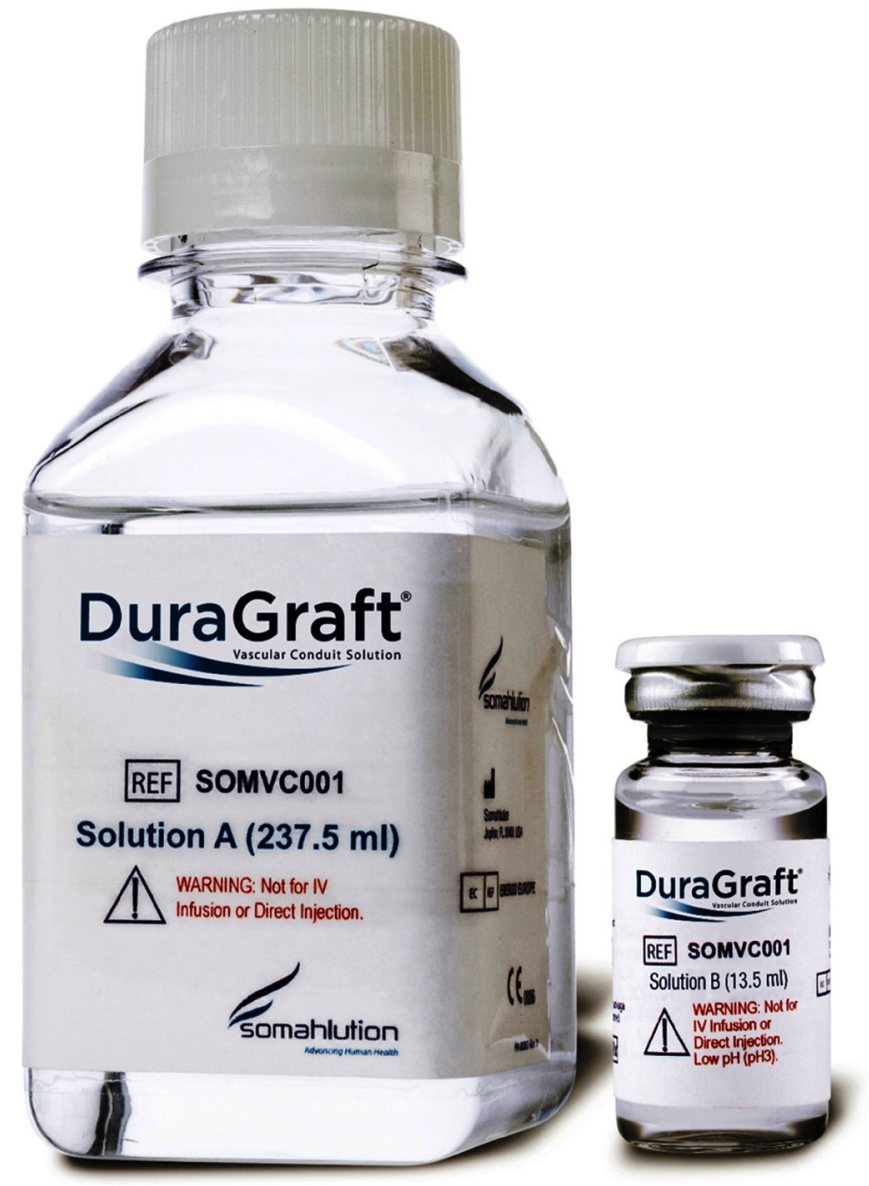

Figure 1 DuraGraft treatment solution.

will be conducted on all patients who withdraw or who are discontinued from the study.

\section{Ethics and informed consent}

All patients provided written informed consent before enrolment into the trial.

\section{Preservation solution}

DuraGraft (SOMVC001) is a specifically designed one-time intraoperative treatment to protect against damage to the structure and function of the vascular endothelium (figure 1). DuraGraft is formulated into an ionically and $\mathrm{pH}$-balanced physiological salt solution containing glutathione, L-ascorbic acid, and L-arginine and other protective ingredients that protect the conduit from the damaging effects of ischaemia (during storage) and handling during CABG.

\section{Conduit harvesting and intraoperative treatment with DuraGraft}

Routinely, all patients will undergo a preoperative evaluation of SVG diameters (luminal diameter) using ultrasound assessment as well as clinical evaluation by the surgeon. Those SVGs that appear to be very atherosclerotic or which have a luminal diameter less than $2 \mathrm{~mm}$ will be excluded prior to surgery. The SVG is harvested using state-of-the-art harvesting (either open or endoscopic) and optimal handling techniques (ie, atraumatic surgical technique, avoidance of over pressurisation during checking for leakage, excessive handling and distortion) in order to reduce traumatic damage to the conduit's endothelium. The SVG segment will be divided into two smaller segments that are used for grafting to the two different target regions (A and B). Each segment will be carefully flushed with and stored within the assigned solution. Storage time from storage to anastomosis is recorded for each of the conduits with a recommended duration of at least $15 \mathrm{~min}$. The SVG segments are only exposed to the assigned preservation solution from the time of harvest until completion of distal and proximal anastomosis and reperfusion.

\section{CABG procedure}

CABG surgery either using on-pump or off-pump techniques (at the discretion of the surgeon) is carried out according to standard and state-of-the-art surgical techniques as described elsewhere. Flow measurements are performed for each conduit and recorded using a hand-held flow metre after reperfusion and prior to chest closure.

\section{MDCT angiography protocol}

The protocol used in this trial for the assessment of wall thickness is based on a validated method by Lau et $a l^{17}$ while for the evaluation of significant stenosis and/or graft occlusion, the Fitzgibbon's scale B and $\mathrm{O}$ is used. MDCT examination is performed with a 64-slice or better scanner (Lightspeed Plus, GE Medical Systems) for angiographic evaluation of grafts as follow-up procedure at 4-6 weeks, 3 months and 12 months (please see online supplementary information for further technical details).

\section{Data collection}

All study information, surgical procedural details, postoperative details, concomitant medications and serious and non-serious adverse events will be collected in the Electronic Data Capture (EDC) system. Site personnel will perform data entry and data corrections in the electronic case report form (eCRF).

Quality control and data validation procedures will be applied to ensure the validity and accuracy of the clinical database.

\section{Primary and secondary endpoints and safety parameters}

The primary short-term endpoint is the magnitude of change between $4-6$ weeks and 3 months post-CABG surgery in mean wall thickness between the paired grafts as determined by 64-slice or better MDCT angiography. The wall thickness will be calculated every $10 \mathrm{~mm}$ for all grafts (varying lengths of the grafts and thus number of measurements used in creating the average for each graft) by subtracting the lumen diameter in millimetre using contrast enhanced CT from the total vessel diameter in millimetre using non-enhanced CT and dividing by two. Each $10 \mathrm{~mm}$ segment is graded for quality, and the mean wall thickness for the whole graft will be computed as the average of all wall thickness measures of acceptable quality for each 


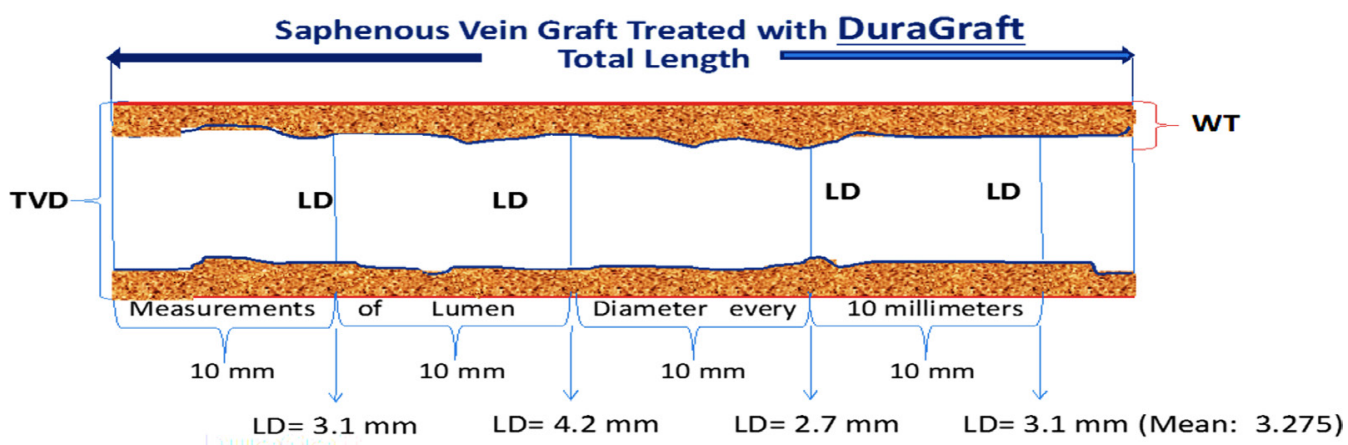

Figure 2 MDCT analysis protocol: each graft was evaluated from proximal to distal in 10 mm intervals. LD, lumen diameter; MDCT, multidetector CT; TVD, total vessel diameter; WT, wall thickness.

of the study grafts at 4-6 weeks and 3 months. The magnitude of change will be calculated per graft as the difference between the 4-6 weeks and the 3-month time points. The magnitude of these changes will then be compared between the paired grafts. The primary long-term endpoint will be the change from 4-6 weeks to 12 months following CABG surgery in lumen diameter calculated as the average of the mean lumen diameter over each graft and the lumen diameter at point of maximal stenosis within graft, using 64-slice or better MDCT angiography (figures 2 and 3).

The secondary efficacy endpoints will assess additional parameters for wall thickness, total lumen diameter, maximum degree of stenosis and others in a paired fashion (DuraGraft-treated SVG vs saline-treated SVG) and also in a longitudinal fashion analysing changes from 4 weeks to 6 weeks and 3 months to 12 months for each graft.
The primary safety endpoint is the incidence of vein graft thrombosis (occlusion). The secondary safety endpoints are (1) incidence of composite safety endpoints consisting of MACE (death, MI and repeat revascularisation), increased angina, arrhythmias, SOB, graft occlusion and significant stenosis based on Fitzgibbon's scale B and $\mathrm{O}$, if determined to be related to specific graft, and (2) incidence of adverse events.

Primary safety endpoints as well as all composite safety endpoints will be adjudicated by an independent blinded review committee to determine if the adverse cardiac events can be attributed to a graft level event.

\section{Statistical considerations and analysis}

Statistical analyses will be performed using SAS V.9.4 or higher. Unless otherwise specified, all statistical tests will be two sided and performed at a significance level of 0.05 . No adjustments for multiple testing will be done. Study

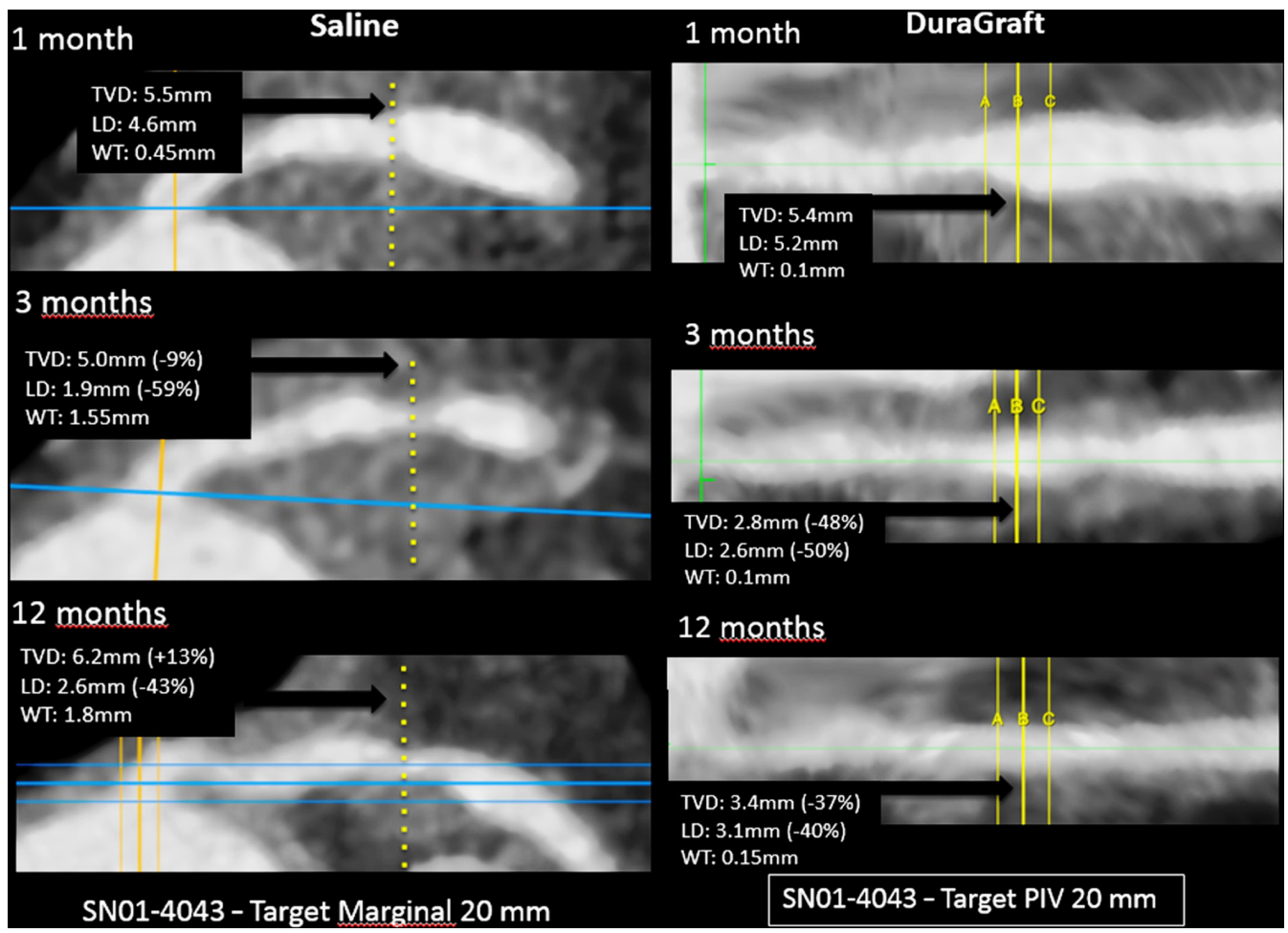

Figure 3 Exemplary primary short-term and long-term endpoint MDCT angiography calculation. LD, lumen diameter; MDCT, multidetector CT; TVD, total vessel diameter; WT, wall thickness. 
variables will be summarised using descriptive statistics. These will include $\mathrm{N}$, mean, median, SD, first (Q1) and third (Q3) quartiles and minimum and maximum for continuous variables. Number of subjects and proportions will be used to summarise categorical variables. Prior to any parametric analyses, basic assumptions of the variable being tested will be checked, and if they are violated, data transformations, non-parametric analyses or other appropriate methods will be performed (such as Wilcoxon signed-rank test). For purposes of data interpretability, the average change score, by treatment group, will be presented, along with the average change in the parameter of interest between the paired grafts.

\section{Efficacy analysis}

Primary and secondary endpoint analysis

The primary endpoint analysis will be based on the evaluable-extension population. Sensitivity analyses will impute missing CT scan data for those not having endpoint CTs. The weighted average lumen diameter will be presented using descriptive statistics by type of solution (DuraGraft vs heparin-dosed saline) and time points (4-6 weeks and 12 months). Changes from 4-6 weeks to 12 months will also be summarised by type of solution. This primary endpoint, change from 4-6 weeks to 12 months in the weighted average lumen diameter, will be compared between DuraGraft graft and heparin-dosed saline graft using a linear mixed model that will include terms for type of solution (DuraGraft vs heparin-dosed saline) and time points (4-6 weeks and 12 months). A factor for target region (left and right) will also be included in the model. This model will account for the correlated nature of the grafts within the patients. The structure of the covariance matrix will be the one yielding the smallest AIC. Contrasts under this longitudinal model will allow for the comparison of the change from 4-6 weeks to 12 months between the two solutions at the 0.05 significance level:

$$
\begin{aligned}
& \mathrm{H}_{0}:\left(\mu_{12} \text { months, SOMVC001 }-\mu_{4-6} \text { weeks, SOMVC001 }\right)- \\
& \left(\mu_{12} \text { months, heparin-dosed saline }-\mu_{4-6} \text { weeks, heparin-dosed saline }\right)=0 \\
& {\left[\mu_{\text {delta }(\text { SOMVC001-heparin }- \text { dosed saline })}=0\right]} \\
& \text { vs. } \\
& \mathrm{H}_{1}:\left(\mu_{12} \text { months, SOMVC001 }-\mu_{4-6} \text { weeks, SOMVC001 }\right)- \\
& \left(\mu_{12} \text { months, heparin-dosed saline }-\mu_{4-6} \text { weeks, heparin-dosed saline }\right) \neq 0 \\
& {\left[\mu_{\text {delta(SOMVC001-heparin-dosed saline })} \neq 0\right. \text { ] }}
\end{aligned}
$$

where $\mu_{4-6 \text { weeks, heparin-dosed saline }}, \mu_{4-6 \text { weeks, somvc001 }}, \mu_{12 \text { months, hepa- }}$ rin-dosed saline and $\mu_{12 \text { months, Somvc001 }}$ are, respectively, the mean of the weighted average lumen diameter in the heparin-dosed saline and DuraGraft solutions at 4-6 weeks and 12 months. In other words, this hypothesis will allow the comparison of the magnitude of the changes between 4-6 weeks and (3 months) 12 months in the paired grafts. For the secondary endpoint analyses, a linear mixed model similar to the one for the primary endpoint will be used.

\section{Sample size calculation and assumptions}

The sample size for this study is based on the literature since little actual information regarding the natural history of wall thickness following bypass has been published. Assuming the null hypothesis of no difference between the two paired grafts, then 90 individuals with randomised grafts to saline or DuraGraft will yield $80 \%$ power using a two-sided type I error of 0.05 against the hypothesis of superiority, assuming an effect size of at least 0.30 [(paired difference in mean wall thickness)/ $\mathrm{SD}$ of the difference]. That is, the DuraGraft preserved grafts will on average have lesser wall thickness than its paired heparin-dosed saline preserved grafts, and if the null hypothesis is rejected, then it may be concluded that DuraGraft preserves the graft better by virtue of the fact that it lessens the increase in wall thickness observed following bypass. To ensure a sufficient evaluable population for this study, at least 100 patients should be enrolled to meet a targeted evaluable population of 90 patients (180 SVGs) and to account for any non-measurable grafts or dropouts, which may occur during the study period. If the missing data exceeds $5 \%$ or the result of Little's test is significant $(\mathrm{P}<0.05)$, then multiple imputations will be used (SAS V.9.4 or higher).

\section{Safety analyses}

Vein graft thrombosis (occlusion) as well as a composite safety endpoint will be assessed using McNemar's test for paired observations displayed in the two-by-two table of the paired graft outcomes. Patency rate will be tested similarly, and descriptive data of the agreement and discordance rates will be presented.

\section{Trial organisation}

The trial is carried out at seven investigational sites (Canada, Denmark and Ireland) and involves a total of 28 different cardiothoracic surgeons. It has a steering committee and independent cardiac adverse event adjudications review committee.

\section{Trial status}

Enrolment of 119 patients was successfully completed on 14 December 2016, and analysis MDCT angiography follow-up is ongoing with an expected completion by the end of 2017 with the completed analysis becoming available by end of the first quarter of 2018 .

\section{Strength and implications of the trial}

VGF still represents a major drawback in CABG impairing long-term clinical outcomes. ${ }^{3}$ Besides surgical trauma that may occur during SVG harvest, in particular the suboptimal preservation of SVGs during storage (prior to anastomosis) has been demonstrated ex vivo to be a key trigger of endothelial damage, which may result in the development of VGD and ultimately failure. ${ }^{3}$

The present trial aims to establish whether the intraoperative treatment of SVGs with DuraGraft has the potential to decrease and/or prevent the occurrence of VGD and VGF by reducing wall thickness increase and/or intima hyperplasia (as early signs of VGD) and the occurrence of SVG stenosis (lumen loss; late signs) in patients undergoing CABG surgery. In addition, it will further provide insight into the longitudinal behaviour of SVGs and the pathophysiology 
Table 1 Study Protocol and short term follow up

\begin{tabular}{|c|c|c|c|c|c|c|c|}
\hline \multirow{3}{*}{$\begin{array}{l}\text { Study period } \\
\text { Study day\procedure }\end{array}$} & \multicolumn{7}{|c|}{ Schedule of events } \\
\hline & \multirow{2}{*}{$\begin{array}{l}\begin{array}{l}\text { Preoperative } \\
\text { screening }\end{array} \\
\text {-10Days }\end{array}$} & \multirow{2}{*}{$\begin{array}{l}\text { Intraoperative } \\
\text { CABG surgery } \\
\text { Day } 1\end{array}$} & \multicolumn{2}{|c|}{ Postoperative } & \multicolumn{2}{|c|}{ Follow-up* } & \multirow[t]{2}{*}{$\begin{array}{l}\text { Early discontinuation } \\
\text { visit }\end{array}$} \\
\hline & & & Day 2 & $\begin{array}{l}\text { Days } 3 \text { to } \\
\text { discharge }\end{array}$ & $\begin{array}{l}\text { Weeks } \\
4-6\end{array}$ & $\begin{array}{l}\text { Month } 3 \\
\text { ( } \pm 1 \text { week) }\end{array}$ & \\
\hline Obtain informed consent $†$ & $x$ & & & & & & \\
\hline $\begin{array}{l}\text { Review inclusion and exclusion } \\
\text { criteria }\end{array}$ & $x$ & & & & & & \\
\hline Medical history & $x$ & & & & & & \\
\hline Demographics & $x$ & & & & & & \\
\hline Prior medications & $x$ & & & & & & \\
\hline Concomitant medications & & $x$ & $\mathrm{x}$ & $x$ & $x$ & $x$ & $x$ \\
\hline 12-lead ECG & $x$ & & $x$ & $x$ & & & \\
\hline Randomisation‡ & & $x$ & & & & & \\
\hline Operative study procedures & & $x$ & & & & & \\
\hline ICU admission & & $x$ & & & & & \\
\hline Measure chest tube blood loss & & $x$ & $x$ & & & & \\
\hline $\begin{array}{l}\text { Cardiac assessments§ } \\
\text { CKMBף }\end{array}$ & & $x$ & $x$ & & & & \\
\hline Troponin & & & $x$ & & & & \\
\hline Glomerular filtration rate (GFR) & & & $x$ & & $\mathrm{x}$ & $x$ & $x$ \\
\hline Vascular flow measurement ${ }^{\star \star}$ & & $x$ & & & & & \\
\hline AEs & & $x$ & $x$ & $x$ & $x$ & $x$ & $x$ \\
\hline MDCT angiography & & & & & $x$ & $x$ & $x$ \\
\hline
\end{tabular}

${ }^{*}$ Follow-up at the weeks 4-6 and month 3 (+1 week) visit must be clinic visits.

†Inform consent must be obtained before any study-related procedures are conducted.

$\ddagger$ Randomisation will occur prior to saphenous vein is harvesting.

§Routine cardiac laboratory testing to be performed as per standard practice. CKMB, and troponin, laboratory results will be collected for the study patients. eGFR will be done.

ПCKMB testing at 8 hours (cay 1), 24 hours (day 2) and 48 hours (day 3 ) will be documented.

${ }^{*}$ Vascular conduit flow will be measured and recorded after reperfusion, prior to chest closure.

$\mathrm{AE}$, adverse Events; $\mathrm{CABG}$, coronary artery bypass grafting; $\mathrm{CKMB}$, creatine kinase kinase myocardial bound; GFR, glomerular filtration rate;

ICU, intensive care unit; MDCT, multidetector CT.

Table 2 Long term follow up

\begin{tabular}{|c|c|c|c|c|}
\hline Study period & $\begin{array}{l}6 \text { (+1 month) } \\
\text { post-CABG } \\
\text { follow-up } \\
\text { telephone-call }\end{array}$ & $\begin{array}{l}9 \text { (+1 month) } \\
\text { post-CABG } \\
\text { follow-up } \\
\text { telephone-call }\end{array}$ & $\begin{array}{l}12 \text { (+1 month) post-CABG } \\
\text { follow-up } \\
\text { clinic visit }\end{array}$ & $\begin{array}{l}\text { Early withdrawal/ } \\
\text { discontinuation visit } \\
\text { clinic visit }\end{array}$ \\
\hline \multicolumn{5}{|l|}{ Procedures/assessments } \\
\hline $\begin{array}{l}\text { Concomitant medications } \\
\text { (cardiovascular) }\end{array}$ & $x$ & $x$ & $\mathrm{X}$ & $x$ \\
\hline AEs & $x$ & $x$ & $x$ & $x$ \\
\hline ECG & & & $x$ & $x$ \\
\hline MDCT angiography & & & $x$ & $x$ \\
\hline
\end{tabular}

Note: informed consent must be obtained for patients who agree for an additional 9-month post-CABG surgery follow-up.

$A E$, adverse events; CABG, coronary artery bypass grafting;GFR, glomerular filtration $r$ a t e; MDCT, multidetector $C T$. 
VGD and VGF. By longitudinal MDCT angiography, the magnitude of change in wall thickness (early signs) at 4-6 weeks and at 3 months post-CABG and the magnitude of change of lumen diameter loss/development of stenosis (late signs) will be evaluated.

Indeed, MDCT angiography is a highly accurate tool to detect stenosis in bypass grafts with sensitivity, specificity, negative and positive predictive values of $97 \%, 97 \%, 93 \%$ and $99 \%$, respectively. ${ }^{18}$ More specifically, 64-slice MDCT has been used to assess graft wall thickness and patency rates as early as 1 month and 12 months after CABG procedures. ${ }^{19}$ MDCT has demonstrated its sensitivity to measure wall thickness and lumen diameter. Lau and colleagues ${ }^{17}$ demonstrated MDCT measure of a non-occluded SVG mean loss of SVG lumen diameter of $9 \%$ $(3.69-3.36 \mathrm{~mm})$ between postoperative months 1 and 12 . Therefore, the 64-slice or better MDCT angiography was selected for this study based on its general ability to assess vessel plaque volume and percentage diameter stenosis with excellent sensitivity and specificity, ${ }^{20}$ including the assessment of SVG wall thickness.

According to Lau et al, intimal hyperplasia as assessed by wall thickening occurs and can be measured within 4-6 weeks post-CABG. This timing is consistent with mechanisms elucidated in animal models of intimal hyperplasia. Furthermore, clinical wall thickening can stabilise or regress sometime between 1 month and 12 months post-CABG. ${ }^{17}$ Interestingly, clinical data on SVGs used for peripheral bypass surgery indicate that wall thickening stabilises at 3 months and begins to regress at 6 months postgrafting. ${ }^{21}$ While indeed wall thickness measurements have been established as meaningful measurements for assessing the risk for development of cardiac events and mortality, the amount of wall thickness or magnitude of change in wall thickness that translates into a clinically meaningful prognostic value remains unclear. In part, this is due to differences in measurement procedures, limited studies assessing early $(<6$ months post-CABG) wall changes, and the error in measurement within a specific imaging methodologies such as MDCT, optical coherence tomography (OCT), Intravascular Ultrasound (IVUS) or others.

Finally, the sophisticated within-patient design used in this trial (with each patient having two graft segments with one immersed in DuraGraft and the other in heparin dosed saline) will facilitate a paired analysis of SVGs within each patient. Importantly, this will allow for a better control of other factors contributing to VGD and VGF within a patient and thus will substantially reduce a potential outcome bias due to patient-specific or conduit-specific variabilities.

\section{Ethics and dissemination}

All patients provided written informed consent before enrolment into the trial. Results will be submitted for publication in a peer-reviewed journal.

\footnotetext{
Author affiliations

${ }^{1}$ Department of Surgery, Montreal Heart Institute, Montréal, Canada

${ }^{2}$ Institut Universitaire de Cardiologie et de Pneumologie, Quebec City, Canada
}

${ }^{3}$ Dept. of Cardiac Surgery, Rigshopitalet University of Copenhagen, Copenhagen, Denmark

${ }^{4}$ Hôpital du Sacré-Coeur, Montréal, Québec, Canada

${ }^{5}$ Somahlution Inc, Jupiter, Florida

${ }^{6}$ Cardiovascular Imaging Service SDN IRCCS, Naples, Italy

${ }^{7}$ University of Alabama, Birmingham, UK

${ }^{8}$ Galway University Hospital, Galway, Ireland

${ }^{9}$ New Brunswick Heart Centre, Saint John, Canada

${ }^{10}$ Clinic for Cardiac Surgery, University Hospital Zurich, Zurich, Switzerland

Contributors The International Committee of Medical Journal Editors (ICMJE) criteria for authorship have been met. TG, VS, FC and LPP designed the study. $\mathrm{PV}, \mathrm{PSO}, \mathrm{NN}, \mathrm{HJ}, \mathrm{FC}, \mathrm{DV}$ and $\mathrm{CB}$ were responsible for collecting and processing data. WBA, TG, GC, MYE and LPP drafted the manuscript. All authors read, revised critically and approved the final manuscript.

Funding This trial is funded from Somahlution Inc, Jupiter, Florida, USA.

Competing interests MYE and LPP are consultants, TG is the CMO and VS is an employee of Somahlution Inc, Jupiter, Florida, USA.

Patient consent Obtained.

Ethics approval The regional ethics committee has approved the trial at each clinical site.

Provenance and peer review Not commissioned; externally peer reviewed. Data sharing statement No additional data are available.

Open Access This is an Open Access article distributed in accordance with the Creative Commons Attribution Non Commercial (CC BY-NC 4.0) license, which permits others to distribute, remix, adapt, build upon this work non-commercially, and license their derivative works on different terms, provided the original work is properly cited and the use is non-commercial. See: http://creativecommons.org/ licenses/by-nc/4.0/

(c) Article author(s) (or their employer(s) unless otherwise stated in the text of the article) 2018. All rights reserved. No commercial use is permitted unless otherwise expressly granted.

\section{REFERENCES}

1. Windecker S, Kolh P, Alfonso F, et al. 2014 ESC/EACTS Guidelines on myocardial revascularization: The Task Force on Myocardial Revascularization of the European Society of Cardiology (ESC) and the European Association for Cardio-Thoracic Surgery (EACTS) Developed with the special contribution of the European Association of Percutaneous Cardiovascular Interventions (EAPCI). Eur Heart J 2014;35:2541-619.

2. Finn SD, Gardin JM, Abrams J, et al. 2012 ACCF/AHA/ACP/AATS/ PCNA/SCAI/STS guideline for the diagnosis and management of patients with stable ischemic heart disease: a report of the American College of Cardiology Foundation/American Heart Association task force on practice guidelines, and the American College of Physicians, American Association for Thoracic Surgery, Preventive Cardiovascular Nurses Association, Society for Cardiovascular Angiography and Interventions, and Society of Thoracic Surgeons. Circulation 2012;126:e354-e471.

3. de Vries MR, Simons KH, Jukema JW, et al. Vein graft failure: from pathophysiology to clinical outcomes. Nat Rev Cardiol 2016;13:451-70.

4. Goldman S, Sethi GK, Holman W, et al. Radial artery grafts vs saphenous vein grafts in coronary artery bypass surgery: a randomized trial. JAMA 2011;305:167-74.

5. Alexander JH, Hafley $\mathrm{G}$, Harrington RA, et al. Efficacy and safety of edifoligide, an E2F transcription factor decoy, for prevention of vein graft failure following coronary artery bypass graft surgery: PREVENT IV: a randomized controlled trial. JAMA 2005;294:2446-54.

6. Harskamp RE, Alexander JH, Schulte PJ, et al. Vein graft preservation solutions, patency, and outcomes after coronary artery bypass graft surgery: follow-up from the PREVENT IV randomized clinical trial. JAMA Surg 2014;149:798-805.

7. Samano N, Geijer H, Liden M, et al. The no-touch saphenous vein for coronary artery bypass grafting maintains a patency, after 16 years, comparable to the left internal thoracic artery: A randomized trial. $J$ Thorac Cardiovasc Surg 2015;150:880-8.

8. Souza DS, Johansson B, Bojö L, et al. Harvesting the saphenous vein with surrounding tissue for CABG provides long-term graft patency comparable to the left internal thoracic artery: results 
of a randomized longitudinal trial. J Thorac Cardiovasc Surg 2006;132:373-8.

9. Woodward LC, Antoniades C, Taggart DP. Intraoperative Vein Graft Preservation: What Is the Solution? Ann Thorac Surg 2016;102:1736-46.

10. Wille T, de Groot H, Rauen U. Improvement of the cold storage of blood vessels with a vascular preservation solution. Study in porcine aortic segments. J Vasc Surg 2008;47:422-31.

11. Thatte HS, Biswas KS, Najiar SF, et al. Multi-photon microscopic evaluation of saphenous vein endothelium and its preservation with a new solution, GALA. Ann Thorac Surg 2003;75:1145-52.

12. Weiss DR, Juchem G, Kemkes BM, et al. Extensive deendothelialization and thrombogenicity in routinely prepared vein grafts for coronary bypass operations: facts and remedy. Int $J$ Clin Exp Med 2009;2:95-113.

13. Wilbring M, Tugtekin SM, Zatschler B, et al. Even short-time storage in physiological saline solution impairs endothelial vascular function of saphenous vein grafts. Eur J Cardiothorac Surg 2011;40:811-5.

14. Wise ES, Hocking KM, Eagle S, et al. Preservation solution impacts physiologic function and cellular viability of human saphenous vein graft. Surgery 2015;158:537-46.

15. Tsakok M, Montgomery-Taylor S, Tsakok T. Storage of saphenous vein grafts prior to coronary artery bypass grafting: is autologous whole blood more effective than saline in preserving graft function? Interact Cardiovasc Thorac Surg 2012;15:720-5.

16. Thatte HS, Khuri SF. The coronary artery bypass conduit: I. Intraoperative endothelial injury and its implication on graft patency. Ann Thorac Surg 2001;72:S2245-S2252.

17. Lau GT, Ridley LJ, Bannon PG, et al. Lumen loss in the first year in saphenous vein grafts is predominantly a result of negative remodeling of the whole vessel rather than a result of changes in wall thickness. Circulation 2006;114(1 Suppl):I-435-0.

18. Ropers D, Ulzheimer S, Wenkel E, et al. Investigation of aortocoronary artery bypass grafts by multislice spiral computed tomography with electrocardiographic-gated image reconstruction. Am J Cardiol 2001;88:792-5.

19. Gao C, Ren C, Li D, et al. Clopidogrel and aspirin versus clopidogrel alone on graft patency after coronary artery bypass grafting. Ann Thorac Surg 2009;88:59-62.

20. Ugolini P, Pressacco J, Lespérance J, et al. Evaluation of coronary atheroma by 64 -slice multidetector computed tomography: Comparison with intravascular ultrasound and angiography. Can J Cardiol 2009;25:641-7.

21. Owens CD, Ho KJ, Conte MS. Lower extremity vein graft failure: a translational approach. Vasc Med 2008;13:63-74. 